\title{
An Alternative to the MVU Estimator to Estimate the Level of DC in AWGN
}

\author{
George K. Agordzo ${ }^{1}$, Adjei H. S. Ammah², Yeboah Derrick ${ }^{3}$, Adikah Israel ${ }^{4}$. \\ ${ }^{1,3}$ School of Information and Electronic Engineering, \\ Zhejiang University of Science and Technology, Hangzhou, Zhejiang Province, CN. \\ ${ }^{2}$ College of Information and Communication Engineering, \\ Southwest University of Science and Technology, Mianyang, Sichuan Province, CN. \\ ${ }^{4}$ School of Science \\ Zhejiang University of Science and Technology, Hangzhou, Zhejiang Province, CN.
}

\begin{abstract}
In statistics, Maximum Likelihood Estimation (MLE) is a method of estimating the parameters of a particular statistical model, finding parameter values that maximize probability, observations, and the parameters are specified. The MLE can be seen as a special case of maximum post-positive estimation (MAP), which includes a uniform preventive distribution of parameters, or as a variant of the MAP that ignores the above and is therefore unregulated. Now let's look at an alternative to the MVU estimator, which is desirable in situations where the minimum variance unbiased (MVU) estimator does not exist or cannot be found, even if it exists. This estimator, which relies on the principle of maximum likelihood, is primarily the common method for obtaining a practical estimator. It has the clear advantage of being a crank turning procedure, which allows you to implement it for complicated estimation problems. A clear advantage of MLE is that it can be found numerically for a given dataset. The safest way to find the MLE is to search the grid, as long as the space between the searches are small enough, we are sure to find the MLE.
\end{abstract}

Keywords: Maximum Likelihood Estimation, minimum variance unbiased, Estimator, Probability Distribution Function.

DOI: $10.7176 / \mathrm{ISDE} / 11-3-05$

Publication date: June $30^{\text {th }} 2020$

\subsection{INTRODUCTION}

The estimator using Maximum Likelihood (MLE) is an important tool for determining the real chances of the hypothetical communication model. A communication channel can also be quite complex and therefore, needs a model to simplify calculations on the decoder's side. The model must be close to the complex communication channel. Numerous standard statistical models are used for this task; Gaussian, Binomial, Exponential, Geometric, Poisson, etc. A standard communication model is chosen based on empirical data.

All these models have unique parameters that have these characteristics. The determination of these parameters for the chosen model is necessary to model the communication channel within reach. In statistics, the Maximum Likelihood Estimator (MLE) is a method for assessing the parameters of a particular statistical model that determines the parameter values that allow observations to be made under certain conditions. MLE can be seen as a special case of the rear maximum estimate with a uniform preventive distribution of parameters or as a variant of that ignores the above and is therefore not regulated. The maximum probability method corresponds to too many estimation methods known in statistics. Assuming that increases are generally distributed with unknown average and variance, average and variance can be estimated with MLE, while only a few samples of the general population are known. MLE would achieve this by taking the average and variance as parameters and finding certain parametric values that make the observed results more likely given the model. In general, the maximum probability method for a fixed dataset and an underlying statistical model selects the value of the model parameters that maximize probability function. Maximum probability (MLE) is an important tool for determining the actual possibilities of the hypothetical communication model. 


\section{System Model and Design}

Since it is clear that each country has its own currency and monetary policy, the possibility of currency integration with other countries must be an explicit decision of the authorities. To be rational, this choice must focus primarily on resolving one or more monetary problems, that is, problems that the country faces in

managing its monetary policy and that it cannot resolve in an optimum manner. Therefore, the timeliness of currency integration in West Africa should be assessed on the basis of a specific and in-depth analysis of the currency problems faced by countries in the region separately and collectively. More attention to be given to the benefits and importance of currency among member states in West Africa if the policy is implemented.

\section{Derivation -Maximum Likelihood Estimation}

Without knowing the previous one or making assumptions about the previous one, we cannot calculate the inverse probability in the equation. 14. However, R. A. Fisher (1912) succeeded in overcoming this by presenting the concept of probability and probable axiom. In many cases, there is no analytical solution and guessing is not practical. As a result, most statistical packages use some type of numerical maximization method. But how exactly do these methods work?

There are at least two situations where GLS and possibly MLE could be justified. First, if information about noise variations is known. This simplicity is a minor virtue that wastes sample information.

Maximum likelihood (ML) is the most popular estimation approach due to its applicability in complicated estimation problems. The basic principle is simple: find the parameter $\theta$ that is the most probable to have generated the data $\mathbf{x}$. The ML estimator is in general not optimal in the minimum variance sense. Neither is it unbiased. However, ML estimator is not a poor estimator: asymptotically it becomes unbiased and reaches the Cramer-Rao Lower bound. Maximum Likelihood Estimator (MLE): A parameter that maximizes the data risk likelihood.

$$
\begin{aligned}
& \boldsymbol{x}[\boldsymbol{n}]=\boldsymbol{A}+\boldsymbol{w}[\boldsymbol{n}], \quad \boldsymbol{n}=\mathbf{0}, \mathbf{1}, \ldots \ldots . \quad \boldsymbol{N}-\mathbf{1}, \quad \text { where } \boldsymbol{w}[\boldsymbol{n}] \sim \boldsymbol{N}(\mathbf{0}, \boldsymbol{A}) \text { and } \boldsymbol{A}>\mathbf{0} \\
& p(x ; A)=\frac{1}{(2 \pi A) \frac{n}{2}} \exp \left[-\frac{1}{2 A} \sum_{n=0}^{N-1}(x[n]-A)^{2}\right] \\
& \frac{\partial 1 n p(x ; A)}{\partial A}=\frac{1}{2 A}+\frac{1}{A} \sum_{n=0}^{N-1}(x[n]-A)+\frac{1}{2 A^{2}} \sum_{n=0}^{N-1}(x[n]-A)^{2}
\end{aligned}
$$

Setting it to Zero gives;

$$
\begin{aligned}
& A^{2}+\mathrm{A}-\frac{1}{N} \sum_{n=0}^{N-1} x^{2}[n]=0 \\
& \mathrm{~A}=-\frac{1}{2} \pm \sqrt{\frac{1}{N} \sum_{n=0}^{N-1} x^{2}[n]+\frac{1}{4}}
\end{aligned}
$$

If the PDF is known, then MLE can be used. With MLE, the unknown parameter is estimated by: $\hat{\theta}=\arg \max _{\theta} P(x ; \theta) \rightarrow$ Find $a \theta$, which miximum the probability.

Where $\underline{\mathbf{x}}$ is the vector of observed data. (N sample).

Asymptotically unbiased: $\lim _{n \rightarrow \infty} E[\hat{\theta}]=\theta$

Asymptotically efficient: $\lim _{n \rightarrow \infty} \operatorname{var}[\hat{\theta}]=C R L B$.

Maximum Likelihood Estimator MLE:

$\mathrm{A}=-\frac{1}{2}-\sqrt{\frac{1}{N} \sum_{n=0}^{N-1} x^{2}[n]+\frac{1}{4}}$

Estimator is biased

$$
\mathrm{E}(\widehat{\mathrm{A}})=\mathrm{E}\left(-\frac{1}{2}+\sqrt{\frac{1}{N} \sum_{n=0}^{N-1} x^{2}[n]+\frac{1}{4}}\right)
$$




$$
\begin{aligned}
& \neq=-\frac{1}{2}+\sqrt{E\left(\frac{1}{N} \sum_{n=0}^{N-1} x^{2}[n]\right)+\frac{1}{4}} \text { for all } A \\
& =-\frac{1}{2}+\sqrt{A+A^{2}+\frac{1}{4}} \\
& =\mathrm{A} .
\end{aligned}
$$

But as $\mathbf{N} \rightarrow \infty,(L L N)$

$\frac{1}{N} \sum_{n=0}^{N-1} x^{2}[n] \rightarrow E\left(x^{2}[n]\right)=A+A^{2}$

$\hat{A}=A$

\section{Numerical Simulation}

We will also need to invoke central limit theorems to establish the asymptotic normality of the gradient of the log likelihood, so as to be able to characterize the MLE itself. We will leave it to more advanced treatises such as Amemiya (1985), Newey and McFadden (1994) to establish specific conditions and fine points that must be assumed to claim the "usual" properties for maximum likelihood estimators.

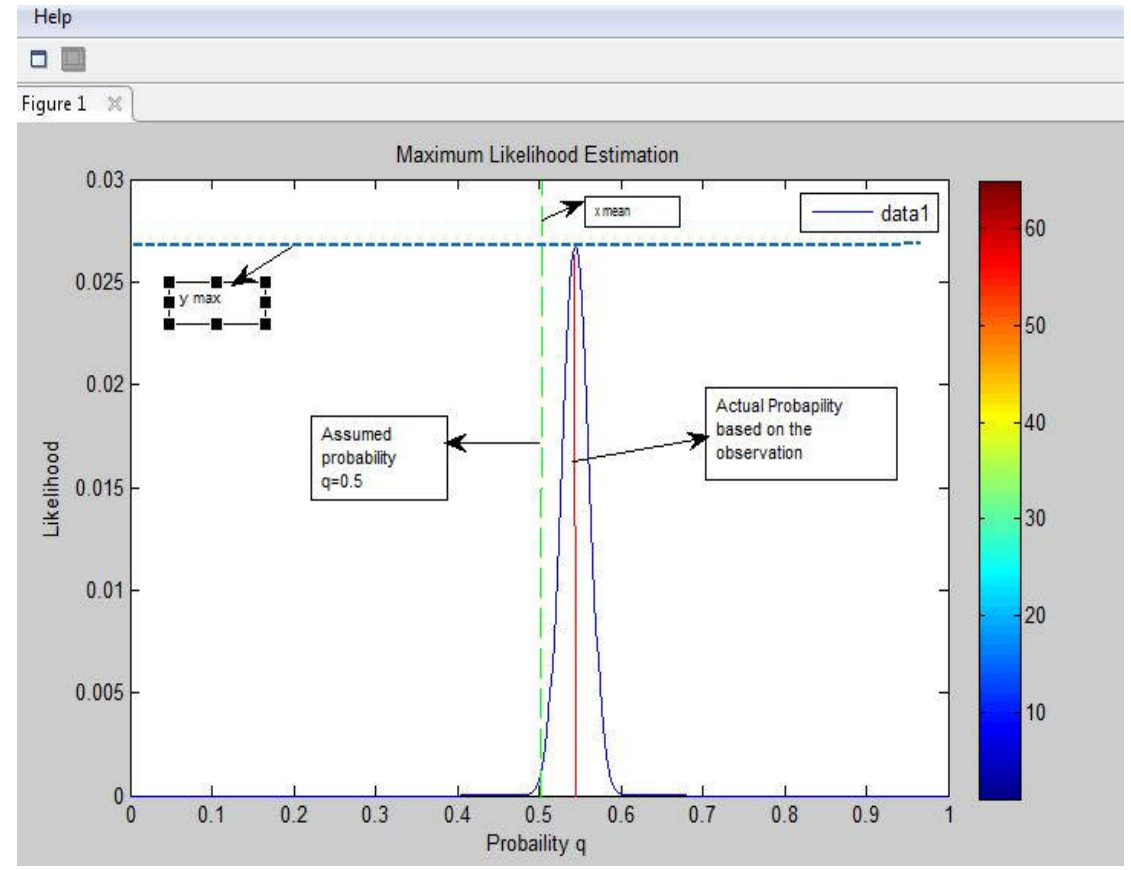

\section{Conclusion}

The MLE always becomes optimal and unbiased as $\mathrm{N} \rightarrow \infty$. Sometimes the MLE is optimal with finite sample sizes, as well. More specifically, if an efficient estimator exists (reaching the CRLB), the MLE will be it. It is observed from the theoretical prove and the simulation above that, the Maximum likelihood Estimator is an alternative to the MVU estimator for Estimation of DC level in AWGN. The maximum probability estimators are consistent, asymptotically normal, and efficiently distributed among the estimators that have these properties. The disadvantage of the technology is that a fully parametric and detailed specification of the data generation process is required. As such, it is prone to incorrect specification issues.

\section{Application}

Modern estimation theory is at the heart of many electronic signal processing systems that have been developed to extract information. Estimating signal processing is essential for many applications. Below are few but not the least of fields or areas where estimations theory applications are useful:

A Radar estimates the echo delay of the received pulse in the presence of noise

A Sonar where the delay in the signal received by each sensor must be estimated in the presence of noise A Speech in which the parameters of the language model are estimated in the presence of speech/speaker variability and ambient noise 
An image in which the position and orientation of an object from a camera image must be estimated in the presence of backlight and noise

A Biomedicine where fetal heartbeats have to be estimated in the presence of sensors and ambient noise.

A Communication in which the carrier frequency of a baseband signal is to be estimated for demodulation in the presence of deteriorating noise

A Control that estimates a motorboat position to correct the navigation correction in the presence of sensor and ambient noise

A Seismology in which the underground extraction of an oil field with strong noise reactions has to be estimated due to the different density of the soil and rock layers.

Most applications require the estimation of an unknown parameter $\theta$ from a set of observation data $\mathrm{x}$ [n], which also contains signals due to sensor inaccuracies, additional noise, signal distortion (convolution noise), model errors, source variability and multiple interruptions (Steven Kay 1993).

\section{Summary}

In summary, the MLE asymptotic PDF is valid in sufficiently large data-sets. The Cramer Rao Lower Bound CRLB can be obtained for signals in case of sound problems, even for short data-sets if the SNR is high enough. Repetition cannot be reduced if the second distraction of protocol probability function is small. The right expression can fluctuate enormously. Even if iteration converges, the point found may not be the global maximum, but only a local maximum or even a local minimum. In general, if the beginning argument is close to the global maximum, the iteration will converge. As the number of observations grows, the MLE is impartial and reaches the CRLB, so it is asymptotically impartial and efficient. But the MLE is not asymptotically equivalent to the MVU; the MLE is distributed asymptotically Gaussian. If there is an objective efficient estimator, the MLE will produce it. For linear models, the MLE is an efficient estimator and the MVU estimator. The MLE is optimal for linear models.

\section{References}

Amemiya, T (1985): Advanced Econometrics, Harvard University Press, Cambridge King, Gary. 1998. Unifying Political Methodology: The Likelihood Theory of Statistical Inference. New York: Cambridge University Press.

Long, J. Scott. 1997. Regression Models for Categorical and Limited Dependent Variables. London: Sage Publications.

Steven M. Kay, Fundamentals of Statistical Signal Processing: Estimation Theory, Prentice-Hall, 1993

Newey, W.K. and D. McFadden (1994),"Large Sample Estimation and Hypothesis Testing," Handbook of Econometrics, Volume 4. 